\title{
A simple strategy to optimally design and manage a photovoltaic plant integrated with a storage system for different applications
}

\author{
Stefano Bracco ${ }^{1}$, Massimo Brignone ${ }^{1}$, Federico Delfino ${ }^{1,2 *}$, Paola Girdinio ${ }^{1}$, Paola Laiolo ${ }^{2}$, Renato Procopio ${ }^{1}$, \\ Mansueto Rossi ${ }^{1}$ \\ ${ }^{1}$ DITEN - Department of Naval, Electrical, Electronic and Telecommunication Engineering \\ ${ }^{2}$ Management Center of Savona Campus facilities \\ University of Genoa \\ Genoa, Italy \\ *federico.delfino@unige.it
}

\begin{abstract}
The main goal of the present paper is that of proposing a methodology for the optimal sizing of a Photovoltaic (PV) unit and a Storage (ST) device, basing on data concerning typical load and $P V$ production profiles. To achieve this result, a set of simple requirements to manage the charging/discharging of the storage is firstly proposed. Then, the overall cost of the whole system is deduced as a function of two variables (PV and ST sizes) and minimized in order to find the optimal sizing of the system. Finally, an economic analysis is presented to determine whether or not the investment is profitable.
\end{abstract}

Keywords-photovoltaic; storage; optimization; planning; management.

\section{INTRODUCTION}

The increase in the exploitation of renewable sources and, among them, of PV generation, is rapidly changing the electric production mix and this, in turn, deeply affects the operating conditions of the power system. The need of mitigating the impact of renewable power plants on distribution networks and the level of "market maturity" reached in several countries by PV technologies have accelerated the trend to "grid parity" for renewable generation $[1,2]$. This has moved the focus from plants primarily intended for power production to be injected into the network to systems devoted to self-consumption and also coupled with storage systems that can bring a new range of application scenarios: renewables integration at grid level, provision of ancillary services, load/generation shifting and peak shaving strategies, etc. As a result, manufacturers are proposing different solutions, targeted also to residential applications, typically composed by a power converter plus a storage system, to be used coupled with renewable generation and eventually gensets.

In the aforesaid framework, advanced control systems to meet the requirements demanded by the grid and the market need to be developed. Moreover, planning strategies have to be set to optimally size and manage distributed generation systems. This is the reason why the literature has recently grown with many works in this direction: many authors evaluate economic indicators to assess the system sustainability [3-5] while others analyze particular economic aspects $[6,7]$ such as the importance of government incentives or key economic policies/strategies.

The present paper proposes a simple but effective way of managing a system consisting of a PV unit and a ST device that is based on a Heuristic Rules System (HRS), able to optimize energy flows within the system. Such Energy Management System is then used to compute the revenue coming from the normal operation of such an integrated system during its whole life, in order to choose the best size of both the PV unit and the ST device. The analysis aims at evaluating the Net Present Value (NPV), the Internal Rate of Return (IRR), the Pay Back Period (PBP) and the Discounted Profitability Ratio (DPR) of the aforesaid investment.

\section{PROBlem DESCRIPTION}

The system here analyzed is composed of the following subsystems: a photovoltaic unit (PV), a storage system (ST), an electrical load (EL) and the connection to the external network (NET). It can be described using the single bus-bar (SBB) model [8]:

$$
P_{N E T, t}+P_{S T, t}+P_{P V, t}+P_{E L, t}=0
$$

where:

- $P_{P V, t}$ is the power injected by the PV unit at time t;

- $P_{S T, t}$ is the power injected by the storage device at time $\mathrm{t}$;

- $P_{N E T, t}$ is the power withdrawn from the external network at time t;

- $P_{E L, t}$ is the power injected (hence negative) by the electric load at time $\mathrm{t}$.

The storage system is represented by: the injected/withdrawn active power, $P_{S T, t}$, the storage energy content, $W_{S T, t}$, and charging/discharging efficiencies [9]. The injected active power depends on $W_{S T, t}$ through a piecewise linear relationship involving two performance coefficients $\eta_{S T, i n}$ 
and $\eta_{S T \text {,out }}$, which are different for the charging and discharging of the battery, as follows:

$$
P_{S T, t}= \begin{cases}\frac{1}{\eta_{S T, i n}} \frac{W_{S T, t-1}-W_{S T, t}}{\Delta t} & W_{S T, t-1}<W_{S T, t} \\ \eta_{S T, \text { out }} \frac{W_{S T, t-1}-W_{S T, t}}{\Delta t} & W_{S T, t-1} \geq W_{S T, t}\end{cases}
$$

Of course, all the involved quantities are subjected to suitable bounds, namely:

$$
W_{S T, \min } \leq W_{S T, t} \leq W_{S T, \max }
$$

where $W_{S T \text {, min }}$ and $W_{S T, \max }$ represent the minimum and the maximum value for the stored energy in the normal operation, respectively; similarly:

$$
P_{S T, c h} \leq P_{S T, t} \leq P_{S T, d s c h}
$$

where $P_{S T, c h}$ and $P_{S T, d s c h}$ represent the maximum power during the charging phase (negative number, according to the aforementioned convention) and the maximum power during the discharging phase (positive number).

The HRS rule here proposed to manage the whole power system can be described as follows:

- if the renewable generation power is greater than the demand and the state of charge of the storage is lower than the maximum, then the battery is charged, otherwise the exceeding produced energy is sold;

- if the renewable generation power is less than the demand and the state of charge of the battery is greater than the minimum, then the battery is discharged and, if it is not sufficient to satisfy the load, power is withdrawn from the grid.

So, combining (1) with the HRS and taking into account the storage device constraints, one can evaluate the power injected by the storage device and by the external network, as follows:

Case A: if $W_{S T, t}<W_{S T, \text { max }}$, then

$$
P_{S T, t}=\max \left\{-\left(P_{P V, t}+P_{E L, t}\right), P_{S T, c h},\left(W_{S T, t-1}-W_{S T, \max }\right) /\left(\eta_{S T, i n} \Delta t\right)\right\}
$$

which follows from the three possible scenarios:

- A.1: the storage can be charged with the difference between the load power and the PV one: $P_{S T, t}=-\left(P_{P V, t}+P_{E L, t}\right)$ without reaching $W_{S T, \max }$ at time $\mathrm{t}+1$;
- A.2: the maximum amount of power that can be stored in the storage device without exceeding $W_{S T, \max }$ at time $\mathrm{t}+1$ is smaller than $\quad-\left(P_{P V, t}+P_{E L, t}\right) ; \quad$ as a consequence, $P_{S T, t}=\left(W_{S T, t-1}-W_{S T, \max }\right) /\left(\eta_{S T, i n} \Delta t\right) ;$

- A.3: $-\left(P_{P V, t}+P_{E L, t}\right)$ is greater than $-P_{S T, c h}$, as a consequence, $P_{S T, t}=P_{S T, c h}$.

The storage state of charge and the power injected by the external network can be evaluated by (2) and (1) as follows:

$$
W_{S T, t+1}=W_{S T, t}-P_{S T, t} \eta_{S T, \text { in }} \Delta t
$$

and

$$
P_{N E T, t}=-P_{S T, t}-P_{P V, t}-P_{E L, t}
$$

Case B: if $W_{S T, t}>W_{S T, \min }$, then

$$
P_{S T, t}=\min \left\{-\left(P_{P V, t}+P_{E L, t}\right), P_{S T, \text { disch }}, \eta_{S T, \text { out }}\left(W_{S T, t-1}-W_{S T, \min }\right) / \Delta t\right\}
$$

which follows from the three possible scenarios:

- B.1: the storage can be discharged with the difference between the load power and the PV one: $P_{S T, t}=-\left(P_{P V, t}+P_{E L, t}\right)$ without reaching $W_{S T, \min }$ at time $\mathrm{t}+1$

- B.2: the maximum amount of power that can be withdrawn from the storage device taking the storage state of charge to $W_{S T, \text { min }}$ at time $\mathrm{t}+1$ is smaller than $-\left(P_{P V, t}+P_{E L, t}\right) ;$ as a consequence, $P_{S T, t}=\eta_{S T, \text { out }}\left(W_{S T, t-1}-W_{S T, \text { min }}\right) / \Delta t$;

- B.3: $-\left(P_{P V, t}+P_{E L, t}\right)$ is greater than $P_{S T, \text { disch }}$, as a consequence, $P_{S T, t}=P_{S T, \text { disch }}$.

Again it follows from (2) and (1) that the storage state of charge and the power injected by the external network are:

$$
W_{S T, t+1}=W_{S T, t}-P_{S T, t} \Delta t / \eta_{S T, \text { out }}
$$

and

$$
P_{N E T, t}=-P_{S T, t}-P_{P V, t}-P_{E L, t}
$$


The HRS can be used to choose the optimal sizes $P_{P V, \max }$ and $W_{S T, \max }$ of both the PV unit and the ST device among the ones available on the market. The choice is done in order to minimize the overall cost/maximize the revenue of the whole system. The overall cost is the sum of the investment, which has to be afforded to buy the two devices, and of the operating cost related to the exchange with the grid.

The cost $C_{0}$ to buy the two devices can be estimated according to the following considerations:

- the storage cost $C_{S T}$ per installed $\mathrm{kWh}$ and the PV cost $C_{P V}$ per installed peak $\mathrm{kW}$ are typically available data;

- the normal life of a PV system is about 20 years and the one of the storage device is about 10 years.

So, one has that:

$$
C_{0}\left(P_{P V, \text { max }}, W_{S T, \text { max }}\right)=C_{P V} P_{P V, \text { max }}+C_{S T} W_{S T, \text { max }}+\frac{C_{S T} W_{S T, \text { max }}}{(1+W A C C)^{9.5}}
$$

which accounts also for the weighted average cost of capital (WACC) in the evaluation of the cost of the second storage device occurring after the first 10 years.

The cost/gain coming from the energy buying/selling from the Distribution System Operator (DSO) in the range $[t \Delta t,(t+1) \Delta t]$ can be evaluated according to the following relation:

$$
C_{N E T, t}\left(P_{P V, \text { max }}, W_{S T, \text { max }}\right)= \begin{cases}C_{\text {sell }, t} P_{N E T, t} \Delta t & P_{N E T, t}<0 \\ C_{\text {buy }, t} P_{N E T, t} \Delta t & P_{N E T, t} \geq 0\end{cases}
$$

being $C_{\text {sell, }, t}$ and $C_{\text {buy,t }}$ the cost, depending on the time slot, of the sold and purchased energy, respectively, and having evaluated $P_{N E T, t}$ according to (7) or (10). The dependence of $C_{N E T, t}$ on $P_{P V, \max }$ and $W_{S T, \max }$ is due to the fact that $W_{S T, \max }$ appears in (5) and (8), thus influencing $P_{S T, t}$, while $P_{P V, \max }$ determines the actual production $P_{P V, t}$ of the PV unit.

The punctual evaluation of (12) over the whole life of the system is of course impossible; to overcome this problem, four typical days have been considered (one for each season of the year) in which the typical PV production (in pu on the PV size $\left.P_{P V, \max }\right)$ and electric load absorption are supposed to be known. This way, repeating each of the four benchmark days 90 times (i.e. the mean duration of each season), one can evaluate the annual cost/gain $C_{N E T, Y}$ of the energy exchange as follows:

$$
C_{N E T, Y}\left(P_{P V, \max }, W_{S T, \text { max }}\right)=90 \sum_{t=1}^{N_{t}} C_{N E T, t}\left(P_{P V, \text { max }}, W_{S T, \text { max }}\right)
$$

having chosen $N_{t}$ s.t. $N_{t} \Delta t$ represents the set of the four aforementioned typical days.

Finally, the total energy cost/gain will be obtained summing $C_{N E T, Y}$ over the 20 years considered, again considering the weighted average cost of capital, as follows:

$$
C_{N E T, \text { life }}\left(P_{P V, \text { max }}, W_{S T, \text { max }}\right)=\sum_{j=1}^{20} \frac{C_{N E T, Y}\left(P_{P V, \text { max }}, W_{S T, \text { max }}\right)}{(1+W A C C)^{j-0.5}}
$$

So, the optimal size of the PV unit and the storage device can be chosen finding the minimum of the following function:

$$
\begin{array}{r}
C\left(P_{P V, \text { max }}, W_{S T, \text { max }}\right)=C_{N E T, \text { life }}\left(P_{P V, \text { max }}, W_{S T, \text { max }}\right)+C_{0}\left(P_{P V, \text { max }}, W_{S T, \text { max }}\right) \\
=C_{N E T, Y}\left(P_{P V, \text { max }}, W_{S T, \text { max }}\right) \frac{(1+W A C C)^{20}-1}{W A C C(1+W A C C)^{19.5}}+ \\
+C_{P V} P_{P V, \text { max }}+C_{S T} W_{S T, \text { max }}\left[1+\frac{1}{(1+W A C C)^{9.5}}\right]
\end{array}
$$

Thanks to the fact that there are only two variables on which to perform the minimum, one can consider the sets $\left\{P_{P V, \max , k}: k=1, \ldots, N_{P V}\right\} \quad$ and $\quad\left\{W_{S T, \max , k}: k=1, \ldots, N_{S T}\right\}$ representing the sizes commercially available, evaluate (15) $N_{P V} N_{S T}$ times in correspondence of any combination of $P_{P V, \max , k}$ and $W_{S T, \max , k}$ and find the minimum value assumed. In the following $P_{P V, \max }^{o p t}$ and $W_{S T, \max }^{o p t}$ will denote the values of the chosen PV and ST sizes, and $C^{\text {opt }}$ the corresponding value of (15).

To demonstrate the economic sustainability of the proposed solution, an economic analysis based on the main investments analysis criteria is now proposed. It should be underlined that (15) has been evaluated in the hypothesis of buying the two storage devices independently.

The solution chosen as the configuration with the lower total cost is compared with the base case (nothing is installed), i.e. $P_{P V, \max }=0$ and $W_{S T, \max }=0$.

This analysis allows to evaluate the proposed technological solution taking into account both the initial investment and future revenues/costs.

The parameters taken into account are:

- Payback Period (PBP)

- Net Present Value (NPV)

- Discounted Profitability Ratio (DPR)

- Internal Rate of Return (IRR)

The $P B P$ is the period of time required to recoup the funds expended in an investment. It can be evaluated using: 


$$
P B P=\min \left\{n: \sum_{s=1}^{n} F C F O_{s}-F C F O_{0} \geq 0\right\}
$$

where:

- $F C F O_{s}$ is the cash flow of year $s$, which, in the present case, corresponds to

$$
\begin{aligned}
F C F O_{S}=\left[C_{N E T, Y}(0,0)-C_{N E T, Y}\left(P_{P V, \text { max }}^{o p t}, W_{S T, \text { max }}^{o p t}\right)\right]+ \\
-\left\{\begin{array}{cr}
0 & s \neq 10 \\
C_{S T} W_{S T, \text { max }}^{o p t} & s=10
\end{array}\right.
\end{aligned}
$$

- $F C F O_{0}$ is the cash flow of year 0 , so it represents the starting investment which is given by:

$$
F C F O_{0}=C_{P V} P_{P V, \text { max }}^{o p t}+C_{S T} W_{S T, \text { max }}^{o p t}
$$

In the case study the solution of equation (16) can be obtained finding the first integer number $\mathrm{n}$ such that:

$$
\begin{gathered}
n\left[C_{N E T, Y}(0,0)-C_{N E T, Y}\left(P_{P V, \text { max }}^{o p t}, W_{S T, \text { max }}^{o p t}\right)\right]+ \\
-\left[C_{P V} P_{P V, \text { max }}+\left\{\begin{array}{ll}
C_{S T} W_{S T, \text { max }}^{o p t} & n<10 \\
2 C_{S T} W_{S T, \text { max }}^{o p t} & n \geq 10
\end{array}\right] \geq 0\right.
\end{gathered}
$$

The NPV measures the excess or shortfall of cash flows, in present value terms, above the cost of funds. It is defined as the algebraic sum of the $F C F O_{s}$ over all the years of the analysis horizon discounted at an interest rate (WACC). In the case study the $N P V$ is:

$$
\begin{aligned}
N P V & =\sum_{s=1}^{20} \frac{F C F O_{s}}{(1+W A C C)^{s-0.5}}-F C F O_{0} \\
& =C_{N E T, Y}(0,0) \frac{(1+W A C C)^{20}-1}{W A C C(1+W A C C)^{19.5}}-C^{o p t}
\end{aligned}
$$

The $D P R$ provides the percentage return of the investment expenditure along the lifetime of the project. It is the ratio between the Net Present Value and the Initial Investment:

$$
\operatorname{DPR}(\%)=100 \frac{N P V}{F C F O_{0}}
$$

In the case study it can be calculated replacing $\mathrm{NPV}$ and $\mathrm{FCFO}_{0}$ respectively with (20) and (18).

The IRR is the rate of return that makes the $N P V$ equal to zero. It can also be defined as the discount rate at which the present value of all future cash flow is equal to the initial investment or in other words the rate at which an investment breaks even. $I R R$ calculations are commonly used to evaluate the desirability of investments or projects. The higher a project's $I R R$ is, the more desirable it is to undertake the project. It can be calculated, starting from (20), as:

$$
\sum_{s=1}^{20} \frac{F C F O_{s}}{(1+I R R)^{s-0.5}}-F C F O_{0}=0
$$

In the case study it can be obtained replacing $F C F O_{s}$ with (17) and $\mathrm{FCFO}_{0}$ with (18). None of these parameters can be sufficient if considered by itself. Only an integrated analysis of all of them can give a complete and appropriate investment evaluation.

\section{RESULTS}

In this section some numerical results are shown considering four possible applications of the proposed system: a residential, a small industry, a medium industry and a large one. They are characterized by different load profiles in the four typical days [3], represented in Fig. 1(a). The PV generation profiles obtained from the irradiance and temperature curves in the same typical days [9] are presented in Fig. 1(b).

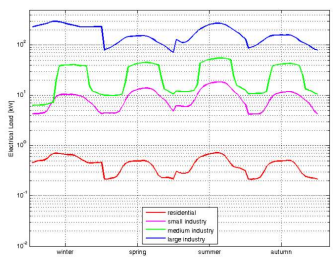

(a)

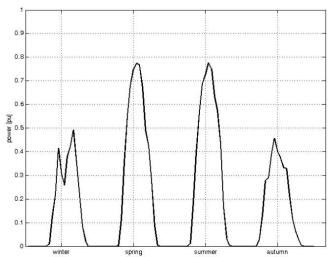

(b)
Fig. 1. Electric load (a) and PV (b) power profiles

The cost of the storage system is assumed equal to 400 $€ / \mathrm{kWh}$ and the cost of the photovoltaic power plant $2500 € / \mathrm{kWp}$. Moreover, the storage system has the following technical characteristics: charging/discharging efficiencies $\eta_{S T, \text { in }}=0.9$ and $\eta_{S T, \text { out }}=0.9$; maximum power in the charging phase $P_{S T, c h}=-(0.3$ $\left.\mathrm{h}^{-1}\right) W_{S T, \max }$; maximum power in the discharging phase $P_{S T, d s c h}=$ $\left(0.3 \mathrm{~h}^{-1}\right) W_{S T, \max }$; depth of Discharge $(D o D)=[10 \%, 100 \%]$, i.e. $W_{S T, \min }=0.1 W_{S T, \max }$. Moreover, according to the Italian electricity market, $C_{\text {sell, }}=0.08 € / \mathrm{kWh}$ and $C_{\text {buy }, t}=0.256 € / \mathrm{kWh}$ if the time index $t$ represents the time slot between 8:00 AM and 8:00 PM, otherwise $C_{b u y, t}=0.198 € / \mathrm{kWh}$. The possible sizes of PV and ST 
are chosen into a discrete set with step of $0.25 \mathrm{~kW}$ and $1 \mathrm{kWh}$, respectively. In the economic analysis, $W A C C=0.035$.

As previously mentioned, the investment economic sustainability cannot be based on a single parameter evaluation, but it is necessary to take into account the economic results favorability for all the economic parameters.

Table 1 Optimal size, overall cost and economical parameters for the four analyzed cases

\begin{tabular}{llllll} 
& unit & residential & small industry & medium industry & large industry \\
\hline$P_{P V, \text { max }}^{\text {opt }}$ & {$[\mathrm{kW}]$} & 1.75 & 40.25 & 132.00 & 680.00 \\
$W_{S T, \text { max }}^{\text {opt }}$ & {$[\mathrm{kWh}]$} & 0.00 & 0.00 & 0.00 & 0.00 \\
$C^{\text {opt }}$ & {$[\mathrm{k} \epsilon]$} & 8.64 & 174.97 & 481.14 & 3575.24 \\
$P B P$ & {$[\mathrm{year}]$} & 8.00 & 8.00 & 8.00 & 8.00 \\
$N P V$ & {$[\mathrm{k} \epsilon]$} & 3.95 & 92.39 & 309.45 & 1383.63 \\
$D P R$ & {$[\%]$} & 90.2 & 91.81 & 93.77 & 81.36 \\
$I R R$ & {$[\%]$} & 12.7 & 12.83 & 13.01 & 11.85 \\
\hline
\end{tabular}

Table 1 reports the overall cost, the optimal size and the calculated economic parameters. More precisely, Table1 shows, for the four analyzed cases, that:

- the $P B P$, which is usually a cut off and allows the investor to understand whether to proceed with the investment analysis or not, is less than half of the plant life, therefore this value allows to go on with the investment analysis;

- the $N P V$ is positive and this means that, actually, the investment allows a generation of gains even if, of course, with different values depending on the considered configuration;

- the $D P R$ is high. This parameter is, perhaps, the most explanatory because it correlates each $N P V$ with the corresponding initial investment and, therefore, it allows to evaluate the investment profitability;

- the IRR is much higher compared to the WACC and, therefore, there is a high level of protection with respect to possible $W A C C$ fluctuations. This means that there is not a potential danger that a $W A C C$ positive fluctuation brings the $N P V$, and then the $D P R$, to zero.

The combined analysis of the four economic parameters allows to state that, for all four case studies, the investment is economically advantageous, because it ensures a high profitability and a high protection level against possible $W A C C$ fluctuations. As it can be easily seen, in accordance with [10], the cost per $\mathrm{kWh}$ of the ST system is still too high in order to make its use profitable. Table 2 presents the relationship between the storage capital cost per installed $\mathrm{kWh}$ and its consequent optimal sizing, highlighting that in order to economically justify the presence of a ST device its cost has to decrease down to $300 € / \mathrm{kWh}$ for the small and medium industry, and down to $200 € / \mathrm{kWh}$ for residential applications.
Table 2 Optimal size of the ST system as function of the cost per $\mathrm{kWh}$

\begin{tabular}{lcccc}
\multicolumn{5}{c}{ small industry } \\
\hline $50 € / k W h$ & $2 \mathrm{kWh}$ & $64 \mathrm{kWh}$ & $151 \mathrm{kWh}$ & $735 \mathrm{kWh}$ \\
$100 € / k W h$ & $2 \mathrm{kWh}$ & $50 \mathrm{kWh}$ & $151 \mathrm{kWh}$ & $735 \mathrm{kWh}$ \\
$150 € / k W h$ & $2 \mathrm{kWh}$ & $50 \mathrm{kWh}$ & $140 \mathrm{kWh}$ & $735 \mathrm{kWh}$ \\
$200 € / k W h$ & $1 \mathrm{kWh}$ & $47 \mathrm{kWh}$ & $133 \mathrm{kWh}$ & $500 \mathrm{kWh}$ \\
$250 € / k W h$ & $0 \mathrm{kWh}$ & $29 \mathrm{kWh}$ & $86 \mathrm{kWh}$ & $420 \mathrm{kWh}$ \\
$300 € / k W h$ & $0 \mathrm{kWh}$ & $9 \mathrm{kWh}$ & $37 \mathrm{kWh}$ & $14 \mathrm{kWh}$ \\
$350 € / k W h$ & $0 \mathrm{kWh}$ & $0 \mathrm{kWh}$ & $0 \mathrm{kWh}$ & $0 \mathrm{kWh}$ \\
$400 \epsilon / k W h$ & $0 \mathrm{kWh}$ & $0 \mathrm{kWh}$ & $0 \mathrm{kWh}$ & $0 \mathrm{kWh}$ \\
$450 \epsilon / k W h$ & $0 \mathrm{kWh}$ & $0 \mathrm{kWh}$ & $0 \mathrm{kWh}$ & $0 \mathrm{kWh}$ \\
\hline
\end{tabular}

\section{CONCLUSION}

The paper presents a simple but effective strategy to manage an integrated system composed of a PV unit and a ST device. Such algorithm has been here used to set up a methodology to choose the optimal sizing of the two devices in four test cases (typical residential load, small industry, medium industry and large industry) each one characterized by a different load profile.

The application of the method, based on the minimization of a function representing the overall cost during the whole life of the system (20 years), has allowed to draw the following conclusions: the actual cost of the storage device is still too high to justify its acquisition in such applications, while the PV optimal size is approximately the one that allows to cover the load peak even in the cold seasons (autumn and winter). Finally, a thorough economic analysis has been conducted to show that, for all four case studies, the investment is economically advantageous, because it ensures a high profitability and a high protection level against possible WACC fluctuations. The economic analysis shows that, for the aforesaid cases, the investment is profitable, but ST systems will be more competitive only if their capital cost diminishes.

\section{REFERENCES}

[1] Castillo-Cagigal M., Caamaño-Martín E., Masa-Bote D., Gutierrez A., Monasterio-Huelin F., Jimenez-Leube J. "PV self-consumption optimization with storage and active DSM for the residential sector", Solar Energy, 2011; 85, (9), pp 2338-38

[2] Femia N., Toledo D., Zamboni W. "Storage unit and load management in photovoltaic inverters for residential application", In Industrial Electronics Society, IECON 2013 - 39th Annual Conference of the IEEE, Vienna, Austria, November 2013, pp 6800-5.

[3] Cucchiella F., D'Adamo I., Koh L.S.C. "Environmental and economic analysis of building integrated photovoltaic systems in Italian regions", Journal of Cleaner Production, 2015, 98, (1), pp 241-252.

[4] Paudel A.M., Sarper H. "Economic analysis of a grid-connected commercial photovoltaic system at Colorado State University-Pueblo", Energy, 2013, 52, pp 289-96.

[5] Peerapong P., Limmeechokchai B. "Investment incentive of grid connected solar photovoltaic power plant under proposed feed-in tariffs framework in Thailand", Energy Procedia, 2014, 52, pp 179-89.

[6] Stevanovic S., Pucar M. "Investment appraisal of a small, grid-connected photovoltaic plant under the Serbian feed-in tariff framework", Renewable and Sustainable Energy Reviews, 2012, 16, pp 1673-82.

[7] Dusonchet L., Telaretti E. "Comparative economic analysis of support policies for solar PV in the most representative EU countries", Renewable and Sustainable Energy Reviews, 2015, 42, pp 986-98. 
[8] Bracco S., Brignone M., Delfino F., Procopio R. "An energy management system for the Savona campus smart polygeneration microgrid", IEEE System Journal, vol. PP, Issue 99, April 2015.

[9] Brekken T.K.A., Yokochi A., Von Jouanne A., Yen Z.Z., Hapke H.M., Halamay D.A. "Optimal energy storage sizing and control for wind power applications", IEEE Transaction Sustainable Energy, 2011, 2, (1), pp 69-77.
[10] RSE. I sistemi di accumulo elettrochimico: prospettive e opportunità (2017), http://anienergia.anie.it/libro-bianco-2-0-i-sistemi-di-accumuloelettrochimico-prospettive-e-opportunita/. 